\title{
Endometrial cancer and microsatellite instability status
}

Abstract: Microsatellite instability (MSI) is an important factor in the development of various cancers as an identifier of a defective DNA mismatch repair system. The objective of our study was to define the association between microsatellite instability status and traditional clinicopathologic characteristics of endometrioid type adenocarcinoma. Material and methods: MSI status of endometrial cancer was examined by employing the Promega MSI Analysis System. This system uses 5 mononucleotide markers to identify MSI in tumour and normal tissue DNA (BAT-25, BAT-26, NR-21, NR-24, and MONO-27), and 2 pentanucleotide markers (Penta C and Penta D) for specimen identification. In this study, we investigated MSI status in 109 endometrial carcinomas. Results and conclusions: One hundred (92\%) of 109 endometrial cancers showed endometrioid type histology and only 9 (8\%) non-endometrioid type. MSI-high was found in 17\% (17/100) of endometrioid type adenocarcinomas, in $0 \%(0 / 9)$ of non-endometrioid carcinomas. Selected clinicopathologic parameters for endometrioid type adenocarcinomas were compared to the MSI status which was separated into two groups - MSI-high and MSI stable. The results showed that MSI-high status was related to clinicopathologic parameters such as deep myometrial invasion and higher histologic grade in endometrioid type adenocarcinomas.

\footnotetext{
*Corresponding author: Daiva Kanopiene, Out Patient Clinic, National cancer institute, Santariskiu 1, LT-08660, Vilnius, Lithuania, E-mail: daiva.kanopiene@nvi.It Jolanta Vidugiriene, Promega Corporation, Madison WI, USA Konstantinas Povilas Valuckas, Radiotherapy and Drug Therapy Center, National cancer institute, Santariskiu 1, LT-08660, Vilnius, Lithuania

Giedre Smailyte, Scientific Research Center, National cancer institute, Santariskiu 1, LT-08660, Vilnius, Lithuania

Saule Uleckiene, Scientific Research Center, National cancer institute, Santariskiu 1, LT-08660, Vilnius, Lithuania Jeff Bacher, Promega Corporation, Madison WI, USA
}

Keywords: Endometrial cancer, Clinicopathologic parameters, Microsatellite instability

DOI 10.1515/med-2015-0005

Received: November 13, 2013; accepted: August 29, 2014

\section{Introduction}

Endometrial cancer is one of the mostly widespread cancers (6th place) among women worldwide, contributing to 290,000 new cases in 2008, and a standardised incidence rate of 8 per 100,000 women [1]. In Europe endometrial cancer is the fourth most frequent cancers in women and the tenth most frequent reason for cancer mortality [2]. This type of cancer almost exclusively develops for women in the post-menopausal period. Its most common histological subtype is adenocarcinoma of endometrioid type or Type I ( $70-80 \%$ of all endometrial cancers) and another, less common, subtype is adenocarcinoma of non-endometrioid type or Type II (20-30\% of all endometrial cancers) $[1,2]$. Type I endometrial cancer is usually hormone sensitive, and occurs in women exposed to estrogens. It is usually well differentiated and has a low potential for myometrial invasion and metastasis [1, 3]. Type II endometrial cancer is not associated with estrogen or progestogen stimulation and arises from atrophic endometrial tissues. It is poorly differentiated, has a high probability of myometrial invasion and metastasis, and a worse prognosis $[4,5]$.

Specific molecular genetic alterations which are thought to be significantly affecting the development of cancer have been identified in several studies of these two different types of endometrial carcinoma [6-8]. One of the molecular alterations associated with endometrioid type (Type I endometrial cancer) is microsatellite instability (MSI), which indicates defects in the DNA mismatch repair system (MMR). Microsatellites are short repetitive nucleotide sequences in the human genome $[9,10]$. Because of 
their repeat structure, microsatellites are prone to slippage or replication errors. Usually, such errors are repaired by the DNA mismatch repair system, however this system can fail resulting in microsatellite instability. Microsatellites exhibit the same number of nucleotide repeats in tumour and healthy tissue of the same individual, however loss of MMR can result in widespread changes in the number of repeat sequences. In 1998, the National Cancer Institute (USA) recommended a panel of 5 mono/or di-nucleotide markers for determination of MSI. The tumour is classified as MSI-high (MSI-H) if it shows instability in at least 2 of 5 markers; MSI-low (MSI-L) if 1 of 5 and microsatellite stable (MSS) if none of 5 [11].

This molecular phenotype was originally found in hereditary non-polyposis colon cancer (HNPCC), also known as Lynch syndrome [12-15]. The most common cancer type related to Lynch syndrome is hereditary colorectal carcinoma, which accounts for 1-3\% of all colorectal cancers and the second most common type is hereditary endometrial cancer, which accounts for $2 \%$ of all endometrial carcinomas [16, 17]. Currently, MSI testing is used when detecting tumours associated with Lynch syndrome as over $90 \%$ of such tumors have high level of microsatellite instability. MSI is a well established and effective genetic marker for detection of Lynch syndrome [18].

MSI status in colorectal carcinomas has been thoroughly studied and it has been found to be important not only for detecting HNPCC, but also as a prognostic and predictive factor in sporadic colorectal carcinomas. MSI has been reported in 15\% of sporadic colorectal carcinomas and was identified as an independent prognostic marker associated with older age, female sex and such clinicopathologic parameters as prevalence in proximal colon, mucinous histology, lower rate of lymph node metastasis, and systemic metastasis. It was also established that MSI tumours have a more favorable prognosis than microsatellite stabile tumours [19, 20]. MSI value as a predictive marker for response to a treatment with 5 -FU (5-fluorouracil), irinotecan and other chemotherapeutic agents is controversial. Studies suggest that stage II colorectal cancers with MSI- high phenotype are irresponsive to 5-FU chemotherapy and MSI-high tumours are more sensitive to treatment with irinotecan than MSS tumours [21, 22].

In endometrial carcinoma, however, the relation between clinicopathologic parameters (which are known to be related with the prognosis of the disease) and MSI status is not yet clear. MSI incidence in sporadic endometrial carcinoma in different studies has been detected in the range from $9 \%$ to $45 \%$ [23-25]. A number of studies have examined the relation between clinicopathologic parameters and MSI status with conflicting results (reasons being small sample size, retrospective analysis, different MSI markers and failure to account for significance of histological type). The majority of published analyses indicate that the MSI-high is more common in endometrioid type histology of endometrial carcinoma than non-endometrioid type [25-27]. It was also stated that MSI-high endometrial tumours were associated with higher tumour grade, deep myometrial invasion and higher clinical stage [26, $28,29]$, whereas the other studies found no relationship between tumour grade, clinical stage and MSI status [23, $24,30]$. None of the studies showed relationship between lower tumour grade and MSI-high and only a few studies observed lower clinical stage in MSI-high endometrial tumours [4]. MSI value as a prognostic factor in sporadic endometrial carcinomas is also not clear. At least one study has found a link between MSI-high status and a higher survival rate [24], in other, though, MSI-high was related with a poor prognosis but only in FIGO I stage of endometrial carcinomas [31]. However, the majority of studies have shown no prognostic advantage [4, 23, 32].

The aim of our study is to investigate whether women age at the time of endometrial cancer diagnosis and selected clinicopathologic parameters such as tumour grade, depth of myometrial invasion and stage of disease found in endometrial cancers are related to MSI-high phenotype. To avoid limitations of other studies and receive conclusive results we have selected only endometrioid type adenocarinomas and used only mononucleotide MSI detection markers.

\section{Material and methods}

\subsection{Patients and selection of tissue}

One hundred nine patients (109 women) who were treated for endometrial carcinoma during the period between April 2010 and June 2011 at the Institute of Oncology, Vilnius University (now National Cancer Institute) were included in the study and written informed consent was obtained from all participants. The Regional Ethical Committee approval was obtained before initiation of this study (protocol No. 158200-05-180-43). All patients were without known familial history of HNPCC (known also as Lynch syndrome) and the history of other cancers. Samples for this study were collected during the surgical treatment and later examined by an expert pathologist. In 
order to histologically classify the endometrial cancers, World Health Organization standards were used and to identify the stage, surgical and pathological data were examined (using the standards of the International Federation of Obstetrics and Gynecology (FIGO) which were established in the year 2010).

\subsection{DNA extraction and MSI analysis}

Specimens of the tumour tissue were extracted from formaline- fixed, paraffin-embedded sample tissue. Tumour tissue DNA was purified by organic extraction, and DNA clean-up was performed. Normal tissue (peripheral blood) DNA was obtained using DNA extraction kit (QIAamp Blood Midi, QIAGEN) according to standard procedures.

The microsatellite phenotype of each endometrial tumour was analysed by employing the Promega MSI Analysis System (version 1.2, Promega Madison, WI, USA). This system uses 5 mononucleotide markers to identify MSI in a tumour and normal tissue DNA (BAT-25, BAT-26, NR-21, NR-24, and MONO-27), and 2 pentanucleotide markers (Penta C and Penta D) to identify whether the tumour and normal DNA specimens are from the same patient. Some studies have shown, that the Promega method is better than the traditional one, which was suggested by the National Cancer Institute in 1998. The reason behind this is that in order to detect MSI-high phenotype, using only mononucleotide markers produce results with higher specifity and better sensivity than using a combination of both mononucleotide and dinucleotide markers, as suggested by the traditional method (2 mononucleotide: BAT-25 and BAT-26, 3 dinucleotide: D2S123, D5S346 and D17S250) [33, 34]. Manufacturer's instructions (Promega) were used to perform the MSI analysis. ABI Prism 3100 Genetic Analyzer (Applied Biosystems) was used to separate the products obtained from fluorescently labeled polymerase chain reaction, and the data were analysed using GeneMapper 3.0 Software (Applied Biosystems).

Tumours were classified by the following method: if more than 2 out of 5 markers demonstrated size alterations or shifts in the tumour DNA with respect to the normal tissue DNA, this cancer was identified as MSIhigh. Tumours with only one marker showing instability were classified as MSI-low while tumours with none of the markers showing instability were classified as microsatellite stable. When doing statistical analysis, MSI-low and microsatellite stable tumours were both regarded as MSI stable.

\subsection{Statistical analysis}

In order to compare MSI-high and MSI stable groups by women age and selected clinicopathologic characteristics (tumour grade, depth of myometrial invasion and stage of disease), the $t$ test and Fisher's exact test were used. Differences were regarded as statistically significant for $\mathrm{p}<0.05$.

\section{Results}

\subsection{MSI analysis in endometrial carcinomas}

The mean age of women at the time of endometrial cancer diagnosis was 64.0 years (range 40-83). 100 of 109 (92\%) endometrial carcinomas showed endometrioid type histology and only 9 (8\%) non-endometrioid types. Microsatellite instability analysis was susccessful for all the tumours of the patients involved in the study with $16 \%$ (17/109) of the cases being classified as MSI-high (Table 1). MSI-high was only found in endometrioid type adenocar-

Table 1: Microsatellite instability (MSI) status according to the histological type of cancer

\begin{tabular}{llll}
\hline Histological type & Number of patients & MSI-high n (\%) & MSI stable n (\%) \\
\hline Total & 109 & $17(15.6 \%)$ & $92(84.4 \%)$ \\
\hline Endometrioid adenocarcinoma & 100 & $17(17.0 \%)$ & $83(83.0 \%)$ \\
\hline Serous adenocarcinoma & 3 & 0 & $3(100 \%)$ \\
\hline Clear cell carcinoma & 1 & 0 & $1(100 \%)$ \\
\hline Adenosarcoma & 5 & 0 & $5(100 \%)$ \\
\hline
\end{tabular}


Table 2: Clinicopathologic characteristics according to the microsatellite instability (MSI) status in endometrioid type adenocarcinomas

\begin{tabular}{|c|c|c|c|c|}
\hline \multirow[t]{2}{*}{ Characteristics } & \multirow[t]{2}{*}{ Number of patients } & \multicolumn{2}{|c|}{ MSI status } & \multirow[t]{2}{*}{$p$ value } \\
\hline & & MSI-high n (\%) & MSI stable n (\%) & \\
\hline Total & 100 & 17 (17\%) & $83(83 \%)$ & \\
\hline \multicolumn{5}{|l|}{ Tumour grade } \\
\hline G 1 & 40 & $2(5 \%)$ & $38(95 \%)$ & $p=0.007$ \\
\hline G $2-3$ & 60 & $15(25 \%)$ & $45(75 \%)$ & \\
\hline \multicolumn{5}{|l|}{ Myometrial invasion } \\
\hline$<50 \%$ & 62 & $7(11 \%)$ & $55(89 \%)$ & $p=0.049$ \\
\hline$\geq 50 \%$ & 38 & $10(26 \%)$ & $28(74 \%)$ & \\
\hline \multicolumn{5}{|l|}{ Pathological tumour $(\mathrm{T})$, stage* } \\
\hline T1 confined to the corpus uteri & 88 & $14(16 \%)$ & $74(84 \%)$ & $p=0.33$ \\
\hline T2-T4 expansion beyond the uterus & 12 & $3(25 \%)$ & $9(75 \%)$ & \\
\hline
\end{tabular}

*T1 - corresponds to FIGO I stage, T2-T4 - FIGO II-IV stage

cinomas: there were 17 out of $100(17 \%)$ cases for endometrioid type and 0 out of $9(0 \%)$ for non-endometrioid type. Note, as the number of non-endometrioid type cancers was so low, reliable statistical conclusions for this type could not be drawn.

\subsection{The clinicopathologic characteristics of MSI-high status}

The relationship of clinicopathologic characteristics with MSI status was analysed only for the endometrioid type adenocarcinomas. The results are shown in the Table 2. Characteristics of each MSI-high sample are shown in Table 3.

The mean age of women with MSI-high tumor phenotype was 65.7 years (range: $53-80$ ) and was not statistically different from women with MSI stable phenotype (63.6 years; range: $40-83)(\mathrm{p}=0.214)$. None of the women in $<50$ years old group (a total of 6 cases) had a MSI-high tumour.

There was a highly statistically significant $(p=0.007)$ association between MSI-high phenotype and a higher tumour grade in endometrioid type adenocarcinomas. The frequency of MSI-high status in tumour grade 1 was
$5 \%(2 / 40)$ as distinct from 25\% (15/60) in grade 2 and 3 combined.

A statistically significant association between deep myometrial invasion and MSI-high status was identified $(\mathrm{p}=0.049)$. In tumours with deep myometrial invasion $(\geq 50 \%)$ the MSI-high phenotype was detected in 10 of the 38 cases $(26 \%)$, whereas in tumours which do not have a myometrial invasion or have a superficial myometrial invasion $(<50 \%)$ only 7 of the 62 cases $(11 \%)$ showed MSIhigh status.

The association between the higher clinical cancer stage and MSI-high status was not statistically significant $(p=0.33)$. However, MSI-high phenotype was more frequent in cases where tumour was expanded beyond the uterus $(3 / 12-25 \%)$ than in cases where tumour was only confined to corpus uteri $(14 / 88-16 \%)$.

\section{Discussion}

In the present study we investigated selected clinical pathological parameters of their association with MSI status in sporadic endometrial carcinomas using mononucleotide MSI detection markers. In our study the statistical relationship between the mean age of women at the 
Table 3: Characteristics of each MSI-high sample in endometrioid type adenocarcinomas

\begin{tabular}{|c|c|c|c|c|c|}
\hline \multirow{2}{*}{ Patient No. } & \multirow{2}{*}{ Age at diagnosis (y) } & \multicolumn{3}{|c|}{ Characteristics } & \multirow{2}{*}{ No. of positive MSI markers } \\
\hline & & Tumour grade (G) & FIGO stage & Myometrial invasion (\%)* & \\
\hline 1 & 71 & $\mathrm{G} 2$ & IA & No & $2 / 5$ \\
\hline 2 & 68 & $\mathrm{G} 2$ & IA & No & $4 / 5$ \\
\hline 3 & 54 & $\mathrm{G} 1$ & IA & No & $3 / 5$ \\
\hline 4 & 74 & $\mathrm{G} 2$ & IA & $<50$ & $4 / 5$ \\
\hline 5 & 72 & G1 & IA & $<50$ & $2 / 5$ \\
\hline 6 & 61 & $\mathrm{G} 2$ & IA & $<50$ & $5 / 5$ \\
\hline 7 & 63 & $\mathrm{G} 2$ & IA & $<50$ & $2 / 5$ \\
\hline 8 & 72 & $\mathrm{G} 2$ & IB & $\geq 50$ & $3 / 5$ \\
\hline 9 & 67 & $\mathrm{G} 2$ & IB & $\geq 50$ & $2 / 5$ \\
\hline 10 & 58 & $\mathrm{G} 2$ & IB & $\geq 50$ & $3 / 5$ \\
\hline 11 & 80 & $\mathrm{G} 2$ & IB & $\geq 50$ & $2 / 5$ \\
\hline 12 & 66 & $\mathrm{G} 2$ & IB & $\geq 50$ & $3 / 5$ \\
\hline 13 & 73 & G3 & IB & $\geq 50$ & $2 / 5$ \\
\hline 14 & 61 & $\mathrm{G} 2$ & IB & $\geq 50$ & $4 / 5$ \\
\hline 15 & 63 & $\mathrm{G} 2$ & IIIC & $\geq 50$ & $4 / 5$ \\
\hline 16 & 53 & G3 & IIIC & $\geq 50$ & $4 / 5$ \\
\hline 17 & 61 & $\mathrm{G} 2$ & IVB & $\geq 50$ & $3 / 5$ \\
\hline
\end{tabular}

*No - invasion only in endometrium

time of endometrial cancer diagnosis and MSI status was not significant. No previous study has reported any relationship between age and MSI status in sporadic endometrial carcinomas [14, 26, 30].

MSI-high was found in $17 \%$ of the endometrioid type adenocarcinomas (17/100), in non- endometrioid carcinomas MSI-high was not detected (0/9). Similar results were reported by the other researchers - MSI-high was detected more frequently in endometrioid histologic type than non-endometrioid type [26, 27]. Ours and previously done studies show that MSI is not involved in the majority of cases of non-endometrioid type adenocarcinomas and thus it can be concluded that in case of sporadic endometrioid carcinoma, MSI is almost exclusive to Type I cancers.

In this study the clinicopathologic factors known to be related with the prognosis of the disease were analysed between MSI-high and MSI stable cases only in the endometrioid type adenocarcinomas. In the present study we found that the deep myometrial invasion and higher histologic tumour grade was significantly related to MSI-high status. This relationship with MSI- high phenotype was reported in the most previous studies [26, 29, 32]. In our study, we also observed relationship between MSI-high phenotype and higher clinical stage. However, this relationship was statistically insignificant, but MSIhigh phenotype was more frequently detected (almost twice) in cases where tumour had spread outside the uterus than in cases where tumour was found only in corpus uteri. It is known that the presence of higher histologic tumour grade, deep myometrial invasion and higher clinical cancer stage are well-known prognostic markers which provide higher recurrence rate and worse survival in endometrial carcinomas. Therefore, it can be suggested that MSI-high phenotype is an unfavourable prognostic marker in sporadic endometrial carcinomas. However, there were many reports where no differences in distribution of MSI-high phenotype by tumour grade, myometrial 
invasion and clinical cancer stage were found [23,24,32]. In contrast to colorectal cancers, it can not be concluded that MSI-high phenotype is an independant prognostic indicator for sporadic endometrioid type adenocarcinoma.

\section{Conclusions}

It was found that MSI-high status is related to endometrioid type adenocarcinoma while none of the non-endometrioid type adenocarcinomas showed MSI-high phenotype. The study identified a statistically significant relationship between MSI-high phenotype and clinicopathologic parameters, such as high histologic grade and deep myometrial invasion in endometrioid type adenocarcinoma. Potential conclusion could be drawn that MSI status is related with unfavorable clinicopathologic prognostic factors for endometrioid type adenocarcinoma, however it is inconclusive whether MSI status is an independant prognostic factor. More reasearch in this field needs to be done with larger sample sizes, using standardised MSI markers to receive more conclusive results.

Acknowledgments: This work was a collaboration between investigators at the Institute of Oncology, Vilnius University (now National Cancer Institute) and Promega Corporation. The microsatellite instability analysis was performed using the Microsatellite Instability Analysis System that is manufactured by Promega Corporation. Drs. Jeff Bacher and Jolanta Vidugiriene are employees of Promega Corporation.

We thank Diana Schveigert and Genovefa Chvatovic for excellent technical support in DNA extraction, Raimundas Meskauskas - for pathological examination of samples, prof. Janina Didziapetriene - for preparation of clinical protocol and coordination of this study.

Conflict of interest: The authors report no conflicts of interest.

\section{References}

[1] Weiderpass E, Labreche F. Malignant tumors of the female reproductive system. Saf Health Work 2012; 3:166-180

[2] Bray F, Loos AH, Oostindier M, Weiderpass E. Geographic and temporal variations in cancer of the corpus uteri: incidence and mortality in pre- and postmenopausal women in Europe. Int J Cancer 2005; 117:123-131

[3] Sherman ME. Theories of endometrial carcinogenesis: a multidisciplinary approach. Mod Pathol 2000; 13:295-308

[4] Basil JB, Goodfellow PJ, Rader JS, Mutch DG, Herzog TJ. Clinical significance of microsatellite instability in endometrial carcinoma. Cancer 2000; 89:1758-1764

[5] Hamilton CA, Cheung MK, Osann K, Chen L, Teng NN, Longacre $\mathrm{TA}$, et al. Uterine papillary serous and clear cell carcinomas predict for poorer survival compared to grade 3 endometrioid corpus cancers. Br J Cancer 2006; 94:642-646

[6] Liu FS. Molecular carcinogenesis of endometrial cancer. Taiwan J Obstet Gynecol 2007; 46:26-32

[7] Tinelli A, Mezzolla V, Leo G, Pisano M, Storelli F, Alemanno G, et al. Microsatellite instability (MSI) as genomic markers in endometrial cancer: toward scientific evidences. Mini Rev Med Chem 2010; 10:1356-1365

[8] Weigelt B, Banerjee S. Molecular targets and targeted therapeutics in endometrial cancer. Curr Opin Oncol 2012; 24:554-563

[9] Ellegren H. Microsatellites: simple sequences with complex evolution. Nat Rev Genet 2004; 5:435-445

[10] Li YC, Korol AB, Fahima T, Nevo E. Microsatellites within genes: structure, function, and evolution. Mol Biol Evol 2004; 21:991-1007

[11] Boland CR, Thibodeau SN, Hamilton SR, Sidransky D, Eshleman JR, Burt RW, et al. A National Cancer Institute Workshop on Microsatellite Instability for cancer detection and familial predisposition: development of international criteria for the determination of microsatellite instability in colorectal cancer. Cancer Res 1998; 58:5248-5257

[12] Meyer LA, Broaddus RR, Lu KH. Endometrial cancer and Lynch syndrome: clinical and pathologic considerations. Cancer Control 2009; 16:14-22

[13] Zaanan A, Meunier K, Sangar F, Flejou JF, Praz F. Microsatellite instability in colorectal cancer: from molecular oncogenic mechanisms to clinical implications. Cell Oncol (Dordr) 2011; 34:155-176

[14] Karamurzin Y, Zeng Z, Stadler ZK, Zhang L, Ouansafi I, Al-Ahmadie HA, et al. Unusual DNA mismatch repair-deficient tumors in Lynch syndrome: a report of new cases and review of the literature. Hum Pathol 2012; 43:1677-1687

[15] Leenen CH, van Lier MG, van Doorn HC, van Leerdam ME, Kooi SG, de Waard J, et al. Prospective evaluation of molecular screening for Lynch syndrome in patients with endometrial cancer $</=70$ years. Gynecol Oncol 2012; 125:414-420

[16] van Lier MG, Wagner A, van Leerdam ME, Biermann K, Kuipers E), Steyerberg EW, et al. A review on the molecular diagnostics of Lynch syndrome: a central role for the pathology laboratory. J Cell Mol Med 2010; 14:181-197

[17] Schneider R, Schneider C, Kloor M, Furst A, Moslein G. Lynch syndrome: clinical, pathological, and genetic insights. Langenbecks Arch Surg 2012; 397:513-525

[18] Bartley AN, Luthra R, Saraiya DS, Urbauer DL, Broaddus RR. Identification of cancer patients with Lynch syndrome: clinically significant discordances and problems in tissue-based mismatch repair testing. Cancer Prev Res (Phila) 2012; 5:320-327 
[19] Popat S, Hubner R, Houlston RS. Systematic review of microsatellite instability and colorectal cancer prognosis. J Clin Oncol 2005; 23:609-618

[20] Boland CR, Goel A. Microsatellite instability in colorectal cancer. Gastroenterology 2010; 138:2073-87 e3

[21] Ribic CM, Sargent DJ, Moore M], Thibodeau SN, French A), Goldberg RM, et al. Tumor microsatellite-instability status as a predictor of benefit from fluorouracil-based adjuvant chemotherapy for colon cancer. N Engl J Med 2003; 349:247-257

[22] Bertagnolli MM, Niedzwiecki D, Compton CC, Hahn HP, Hall M, Damas B, et al. Microsatellite instability predicts improved response to adjuvant therapy with irinotecan, fluorouracil, and leucovorin in stage III colon cancer: Cancer and Leukemia Group B Protocol 89803. J Clin Oncol 2009; 27:1814-1821

[23] MacDonald ND, Salvesen HB, Ryan A, Iversen OE, Akslen LA, Jacobs IJ. Frequency and prognostic impact of microsatellite instability in a large population-based study of endometrial carcinomas. Cancer Res 2000; 60:1750-1752

[24] Maxwell GL, Risinger JI, Alvarez AA, Barrett JC, Berchuck A. Favorable survival associated with microsatellite instability in endometrioid endometrial cancers. Obstet Gynecol 2001; 97:417-422

[25] Goodfellow PJ, Buttin BM, Herzog TJ, Rader JS, Gibb RK, Swisher E, et al. Prevalence of defective DNA mismatch repair and MSH6 mutation in an unselected series of endometrial cancers. Proc Natl Acad Sci U S A 2003; 100:5908-5913

[26] An HJ, Kim KI, Kim JY, Shim JY, Kang H, Kim TH, et al. Microsatellite instability in endometrioid type endometrial adenocarcinoma is associated with poor prognostic indicators. Am J Surg Pathol 2007; 31:846-8453

[27] Black D, Soslow RA, Levine DA, Tornos C, Chen SC, Hummer AJ, et al. Clinicopathologic significance of defective DNA mismatch repair in endometrial carcinoma. J Clin Oncol 2006; 24:1745-1753
[28] Baldinu P, Cossu A, Manca A, Satta MP, Pisano M, Casula M, et al. Microsatellite instability and mutation analysis of candidate genes in unselected sardinian patients with endometrial carcinoma. Cancer 2002; 94:3157-3168

[29] Hirasawa A, Aoki D, Inoue J, Imoto I, Susumu N, Sugano K, et al. Unfavorable prognostic factors associated with high frequency of microsatellite instability and comparative genomic hybridization analysis in endometrial cancer. Clin Cancer Res 2003; 9:5675-5682

[30] Buttin BM, Powell MA, Mutch DG, Rader JS, Herzog T], Gibb RK, et al. Increased risk for hereditary nonpolyposis colorectal cancer-associated synchronous and metachronous malignancies in patients with microsatellite instability-positive endometrial carcinoma lacking MLH1 promoter methylation. Clin Cancer Res 2004; 10:481-490

[31] Steinbakk A, Malpica A, Slewa A, Gudlaugsson E, Janssen $E A$, Arends $M$, et al. High frequency microsatellite instability has a prognostic value in endometrial endometrioid adenocarcinoma, but only in FIGO stage 1 cases. Cell Oncol (Dordr) 2011; 34:457-465

[32] Zighelboim I, Goodfellow PJ, Gao F, Gibb RK, Powell MA, Rader JS, et al. Microsatellite instability and epigenetic inactivation of MLH1 and outcome of patients with endometrial carcinomas of the endometrioid type. J Clin Oncol 2007; 25:2042-2048

[33] Murphy KM, Zhang S, Geiger T, Hafez MJ, Bacher J, Berg KD, et al. Comparison of the microsatellite instability analysis system and the Bethesda panel for the determination of microsatellite instability in colorectal cancers. J Mol Diagn 2006; 8:305-311

[34] Wong YF, Cheung TH, Lo KW, Yim SF, Chan LK, Buhard O, et al. Detection of microsatellite instability in endometrial cancer: advantages of a panel of five mononucleotide repeats over the National Cancer Institute panel of markers. Carcinogenesis 2006; 27:951-955 